\title{
Transcendental manifolds in real projective space and Stiefel-Whitney classes
}

\author{
WOJCIECH KUCHARZ AND JOOST VAN HAMEL
}

\begin{abstract}
It is shown that the Stiefel-Whitney classes of a smooth manifold can give obstructions to realizing this manifold as the set of real points of a nonsingular real algebraic subvariety of projective space of a given dimension, even when the manifold can be embedded as an algebraic subset of real projective space of that dimension (meaning that the corresponding real algebraic variety must have complex singularities outside the real points). This strengthens earlier results by Akbulut and King. The result is an application of more technical results concerning algebraic cycles on real varieties combined with the Barth-Larsen Theorem in complex geometry.
\end{abstract}

Mathematics Subject Classification (2000): 14P05 (primary); 14C25, 57R20 (secondary).

\section{Introduction}

Throughout this paper by a real variety $X$ we mean an integral projective scheme over $\mathbb{R}$. We write $X(\mathbb{R})$ for its set of real points, and $X(\mathbb{C})$ for its set of complex points. If $X$ is nonsingular, then either $X(\mathbb{R})$ is empty, or it is a compact smooth $\left(\mathcal{C}^{\infty}\right)$ manifold of dimension equal to that of $X$. Note that there is no (partial) converse: if $X(\mathbb{R})$ is a smooth manifold of dimension equal to that of $X$, then $X$ may still be singular. This is of course in sharp contrast with the fact that $X$ is nonsingular if and only if the complex analytic set $X(\mathbb{C})$ is a complex analytic manifold. The difference between $X(\mathbb{R})$ being smooth and $X$ being nonsingular will play a crucial role in the paper.

A classical result asserts that any nonsingular real variety of dimension $d$ can be embedded in real projective $n$-space $\mathbb{P}^{n}$ (regarded as a real variety), provided $n \geq 2 d+1$. The existence of an embedding in $\mathbb{P}^{n}$ with $n \leq 2 d$ is in general a subtle problem. In the present paper we will see how the topology of the set of real points $X(\mathbb{R})$ sometimes gives restrictions on the minimal $n$ for which $X$ admits an embedding in $\mathbb{P}^{n}$. 
According to the Nash-Tognoli Theorem ( $c f$. Remark 2.7), each compact positive-dimensional smooth manifold $M$ is diffeomorphic to $X(\mathbb{R})$ for some nonsingular real variety $X$; such a variety $X$ is called an algebraic model of $M$. Given a positive integer $n$, we say that $M$ is $n$-transcendental if $M$ admits a smooth embedding into $\mathbb{P}^{n}(\mathbb{R})$, but no algebraic model of $M$ can be embedded in $\mathbb{P}^{n}$. Note that $M$ can only be $n$-transcendental if $n$ is relatively small compared to the dimension of $M$ : the projective embedding theorem mentioned above implies that $M$ will never be $n$-transcendental for $n \geq 2 \operatorname{dim} M+1$. Let us also mention that $M$ cannot be $n$ transcendental if it is embeddable in $\mathbb{P}^{n}(\mathbb{R})$ as an intersection of $\mathcal{C}^{\infty}$ hypersurfaces in general position ( $c f$. [3, Theorem 7.1]).

In the present paper we identify a large class of $n$-transcendental manifolds, many of which can even be realized as nonsingular irreducible algebraic subsets of $\mathbb{P}^{n}(\mathbb{R})$. This strengthens in particular the result of Akbulut and King in [2], where they prove the existence of a nonsingular algebraic subset of $\mathbb{P}^{n}(\mathbb{R})$ that is not isotopic to the set of real points of any nonsingular closed subvariety of $\mathbb{P}^{n}$ $(c f$. also $[1,13])$.

Before stating our result in a precise form, we introduce some notation and terminology. Given a smooth manifold $M$, we denote by $w_{i}(M)$ its $i$ th StiefelWhitney class. The unit $l$-sphere will be denoted by $S^{l}$ :

$$
S^{l}=\left\{\left(y_{i}, \ldots, y_{l+1}\right) \in \mathbb{R}^{l+1}: y_{1}^{2}+\cdots+y_{l+1}^{2}=1\right\} .
$$

We identify $\mathbb{R}^{n}$ with the standard hyperplane complement in $\mathbb{P}^{n}(\mathbb{R})$, i.e., its image under the map

$$
\mathbb{R}^{n} \rightarrow \mathbb{P}^{n}(\mathbb{R}),\left(x_{1}, \ldots, x_{n}\right) \mapsto\left(1: x_{1}: \cdots: x_{n}\right) .
$$

Theorem 1.1. Let $M$ be a compact smooth manifold of dimension $m \geq 1$.

(i) Assume that for some integer $k \geq 2$ there is no cohomology class $\omega \in$ $H^{1}(M, \mathbb{Z} / 2 \mathbb{Z})$ such that for every $i \leq k$ the Stiefel-Whitney class $w_{i}(M) \in$ $H^{i}(M, \mathbb{Z} / 2 \mathbb{Z})$ is a scalar multiple of $\omega^{i}$. Then for all nonnegative integers $l, n$ such that

$$
2 m+2 l-2 k \geq n \geq 2 m+l,
$$

the manifold $M \times S^{l}$ is $n$-transcendental.

(ii) For all nonnegative integers $l, n$ such that

$$
n \geq 2 m+l+1,
$$

there is a smooth embedding of $M \times S^{l}$ in $\mathbb{R}^{n}$ which maps $M \times S^{l}$ onto a nonsingular irreducible algebraic subset of $\mathbb{P}^{n}(\mathbb{R})$.

It is easy to give a concrete example illustrating Theorem 1.1. 
Example 1.2. Let $M$ be a smooth manifold homeomorphic to the complex projective plane. Since $w_{2}(M) \neq 0$ and $H^{1}(M, \mathbb{Z} / 2 \mathbb{Z})=0$, Theorem 1.1 implies that for any integer $l \geq 12$ and any $n$ such that

$$
2 l-4 \geq n \geq l+8
$$

the manifold $M \times S^{l}$ is $n$-transcendental. If, moreover, $n \geq l+9$ (hence $l \geq 13$ ), there is a smooth embedding of $M \times S^{l}$ in $\mathbb{R}^{n}$ which maps $M \times S^{l}$ onto a nonsingular algebraic subset of $\mathbb{P}^{n}(\mathbb{R})$.

In view of the fact that for some of the $n$-transcendental manifolds identified in Theorem 1.1 we do not know whether they can be embedded as an algebraic subset of $\mathbb{P}^{n}(\mathbb{R})$, we consider it an interesting open problem to decide whether every smooth submanifold of $\mathbb{P}^{n}(\mathbb{R})$ is isotopic to a nonsingular algebraic subset of $\mathbb{P}^{n}(\mathbb{R})$.

Theorem 1.1 is proved in Section 2 as an application of our results concerning cycles on real varieties and the Barth-Larsen theorem in complex geometry. In order not to interrupt the flow of exposition, the proof of Theorem 2.1 is postponed until Section 3.

ACKNOWLEDGEMENTS. This paper originated from a conversation between the authors during the Oberwolfach workshop "Reelle Algebraische Geometrie", and was written while the first author was visiting the Max-Planck-Institut für Mathematik in Bonn. The authors would like to thank the Mathematisches Forschungsinstitut Oberwolfach for the support and excellent research environment, and the first author would like to thank the Max-Planck-Institut for the support and hospitality.

\section{Real cycles}

Let $X$ be a nonsingular $n$-dimensional real variety with $X(\mathbb{R})$ nonempty. We have the cycle map

$$
\mathrm{cl}_{\mathbb{R}}: \mathcal{Z}^{k}(X) \rightarrow H^{k}(X(\mathbb{R}), \mathbb{Z} / 2 \mathbb{Z}),
$$

defined on the group $\mathcal{Z}^{k}(X)$ of algebraic cycles on $X$ of codimension $k$ : for any closed subvariety $V$ of $X$ of codimension $k$, the cohomology class $\mathrm{cl}_{\mathbb{R}}(V)$ is Poincaré dual to the homology class in $H_{n-k}(X(\mathbb{R}), \mathbb{Z} / 2 \mathbb{Z})$ represented by $V(\mathbb{R})$ if $\operatorname{dim} V(\mathbb{R})=$ $n-k\left(c f .[6]\right.$ or $[4,5]$ for the definition of this homology class) and $\operatorname{cl}_{\mathbb{R}}(V)=0$ if $\operatorname{dim} V(\mathbb{R})<n-k$. We concentrate our attention on the image

$$
H_{\mathrm{alg}}^{k}(X(\mathbb{R}), \mathbb{Z} / 2 \mathbb{Z})=\operatorname{cl}_{\mathbb{R}}\left(\mathcal{Z}^{k}(X)\right)
$$

of $\mathcal{Z}^{k}(X)$ under $\mathrm{cl}_{\mathbb{R}}$ ( $c f$. [5] for a short survey of its properties and applications). It is well-known that each Stiefel-Whitney class $w_{k}(X(\mathbb{R}))$ is in $H_{\text {alg }}^{k}(X(\mathbb{R}), \mathbb{Z} / 2 \mathbb{Z})$ 
(cf. [6]). What other cohomology classes are in $H_{\mathrm{alg}}^{k}(X(\mathbb{R}), \mathbb{Z} / 2 \mathbb{Z})$, depends very much on the algebraic-geometric properties of $X$.

The groups $H_{\text {alg }}^{k}(-, \mathbb{Z} / 2 \mathbb{Z})$ have the expected functorial property: if $f: X \rightarrow$ $Y$ is a morphism of nonsingular real varieties, the smooth map

$$
f(\mathbb{R}): X(\mathbb{R}) \rightarrow Y(\mathbb{R})
$$

determined by $f$ satisfies

$$
f(\mathbb{R})^{*}\left(H_{\text {alg }}^{k}(Y(\mathbb{R}), \mathbb{Z} / 2 \mathbb{Z})\right) \subset H_{\text {alg }}^{k}(X(\mathbb{R}), \mathbb{Z} / 2 \mathbb{Z})
$$

for $k \geq 0$ (cf. [6]).

Much more surprising is the fact that if we allow ourselves strong conditions on the topology of the holomorphic map

$$
f(\mathbb{C}): X(\mathbb{C}) \rightarrow Y(\mathbb{C}),
$$

we may actually bound $H_{\mathrm{alg}}^{k}(X(\mathbb{R}), \mathbb{Z} / 2 \mathbb{Z})$ from above as subgroup in

$$
H^{k}(X(\mathbb{R}), \mathbb{Z} / 2 \mathbb{Z})
$$

by the image of the cohomology of $Y(\mathbb{R})$ under the pull-back via $f(\mathbb{R})$. This is the content of the result below, which plays a crucial role in the proof of Theorem 1.1.

Theorem 2.1. Let $f: X \rightarrow Y$ be a morphism of nonsingular real varieties having real points. If $r \geq 0$ is such that

$$
f(\mathbb{C})^{*}: H^{j}(Y(\mathbb{C}), \mathbb{Z}) \rightarrow H^{j}(X(\mathbb{C}), \mathbb{Z})
$$

is an isomorphism for all $j \leq r$, then

$$
H_{\text {alg }}^{k}(X(\mathbb{R}), \mathbb{Z} / 2 \mathbb{Z}) \subset f(\mathbb{R})^{*}\left(H^{k}(Y(\mathbb{R}), \mathbb{Z} / 2 \mathbb{Z})\right)
$$

for all $k \leq r / 2$.

\section{Remark 2.2.}

(i) For the case $r=2$ we have in fact that if

$$
f(\mathbb{C})^{*}: H^{j}(Y(\mathbb{C}), \mathbb{Z}) \rightarrow H^{j}(X(\mathbb{C}), \mathbb{Z})
$$

is an isomorphism for all $j \leq 2$, then

$$
H_{\text {alg }}^{1}(X(\mathbb{R}), \mathbb{Z} / 2 \mathbb{Z})=f(\mathbb{R})^{*}\left(H_{\text {alg }}^{1}(Y(\mathbb{R}), \mathbb{Z} / 2 \mathbb{Z})\right)
$$

for $k=1$ (and $k=0$ as well, but this is trivial). 
This sharper result, not used in the present paper, is in fact much easier to prove than the general case, and should already be known implicitly or explicitly by all experts, although we are not aware of an explicit occurrence of this result in the literature. Indeed, the hypothesis immediately implies that

$$
f_{\mathbb{C}}^{*}: \operatorname{Pic}\left(Y_{\mathbb{C}}\right) \rightarrow \operatorname{Pic}\left(X_{\mathbb{C}}\right)
$$

is an isomorphism of the Picard groups of the associated complex varieties, from which it easily follows that

$$
f^{*}: \operatorname{Pic}(Y) \rightarrow \operatorname{Pic}(X)
$$

is an isomorphism, which implies the conclusion on $H_{\mathrm{alg}}^{1}$, since the cycle map $\mathrm{cl}_{\mathbb{R}}$ is compatible with rational equivalence of algebraic cycles.

(ii) If $Y=\mathbb{P}^{n}$ in Theorem 2.1, then

$$
H_{\mathrm{alg}}^{k}(X(\mathbb{R}), \mathbb{Z} / 2 \mathbb{Z})=f(\mathbb{R})^{*}\left(H_{\mathrm{alg}}^{k}\left(\mathbb{P}^{n}(\mathbb{R}), \mathbb{Z} / 2 \mathbb{Z}\right)\right)=f(\mathbb{R})^{*}\left(H^{k}\left(\mathbb{P}^{n}(\mathbb{R}), \mathbb{Z} / 2 \mathbb{Z}\right)\right)
$$

for all $k \leq r / 2$, since $H_{\text {alg }}^{q}\left(\mathbb{P}^{n}(\mathbb{R}), \mathbb{Z} / 2 \mathbb{Z}\right)=H^{q}\left(\mathbb{P}^{n}(\mathbb{R}), \mathbb{Z} / 2 \mathbb{Z}\right)$ for all $q \geq 0$.

We postpone the proof of Theorem 2.1 until Section 3. Presently we derive consequences of Theorem 2.1 and some classical results from complex geometry.

By combining Theorem 2.1 with the Lefschetz theorem for complete intersections, we get:

Corollary 2.3. Let $X$ be a nonsingular complete intersection in $\mathbb{P}^{n}$ with $X(\mathbb{R})$ nonempty, and let $i: X \hookrightarrow \mathbb{P}^{n}$ be the inclusion morphism. Then

$$
H_{\mathrm{alg}}^{k}(X(\mathbb{R}), \mathbb{Z} / 2 \mathbb{Z})=i(\mathbb{R})^{*}\left(H^{k}\left(\mathbb{P}^{n}(\mathbb{R}), \mathbb{Z} / 2 \mathbb{Z}\right)\right)
$$

for all $k \leq(\operatorname{dim} X-1) / 2$.

Proof. By the Lefschetz Theorem (see for example [15, Example 3.1.31]), the restriction map

$$
i(\mathbb{C})^{*}: H^{j}\left(\mathbb{P}^{n}(\mathbb{C}), \mathbb{Z}\right) \rightarrow H^{j}(X(\mathbb{C}), \mathbb{Z})
$$

is an isomorphism for $j \leq \operatorname{dim} X-1$, and hence it suffices to apply Theorem 2.1 via Remark 2.2.(ii).

Similarly, Theorem 2.1 combined with the Barth-Larsen theorem yields the following result, which plays an essential role in the proof of Theorem 1.1.

Corollary 2.4. Let $X$ be a nonsingular closed subvariety of $\mathbb{P}^{n}$ with $X(\mathbb{R})$ nonempty, and let $i: X \hookrightarrow \mathbb{P}^{n}$ be the inclusion morphism. Then

$$
H_{\mathrm{alg}}^{k}(X(\mathbb{R}), \mathbb{Z} / 2 \mathbb{Z})=i(\mathbb{R})^{*}\left(H^{k}\left(\mathbb{P}^{n}(\mathbb{R}), \mathbb{Z} / 2 \mathbb{Z}\right)\right)
$$

for all $k \leq \operatorname{dim} X-(n / 2)$. In particular,

$$
w_{k}(X(\mathbb{R})) \in i(\mathbb{R})^{*}\left(H^{k}\left(\mathbb{P}^{n}(\mathbb{R}), \mathbb{Z} / 2 \mathbb{Z}\right)\right)
$$

for all $k \leq \operatorname{dim} X-(n / 2)$. 
Proof. By the Barth-Larsen theorem ([14], or see [15]), the restriction map

$$
i(\mathbb{C})^{*}: H^{j}\left(\mathbb{P}^{n}(\mathbb{C}), \mathbb{Z}\right) \rightarrow H^{j}(X(\mathbb{C}), \mathbb{Z})
$$

is an isomorphism for $j \leq 2 \operatorname{dim} X-n$, and hence it suffices to apply Theorem 2.1 via Remark 2.2.(ii).

There are many Barth-Larsen type theorems available in the literature ( $c f .[15$, Theorem 3.2.1] and the references cited there). They can be used in conjunction with Theorem 2.1 to obtain the correspoonding versions of Corollary 2.4. We leave the details to the interested reader.

The following result gives in certain cases a strengthening of the second part of Corollary 2.4, but it will not be used in the rest of the paper.

Corollary 2.5. Let $X$ be a nonsingulaar closed subvariety of $\mathbb{P}^{n}$ with $X(\mathbb{R})$ nonempty, and let $i: X \hookrightarrow \mathbb{P}^{n}$ be the inclusion morphism. If $3 n \leq 4 \operatorname{dim} X$, then

$$
w_{k}(X(\mathbb{R})) \in i(\mathbb{R})^{*}\left(H^{k}\left(\mathbb{P}^{n}(\mathbb{R}), \mathbb{Z} / 2 \mathbb{Z}\right)\right)
$$

for all $k \geq 0$.

Proof. Our aim is to show that, under the hypotheses, the total Stiefel-Whithey class $w(X(\mathbb{R}))$ is contained in the subring

$$
R:=i(\mathbb{R})^{*}\left(H^{*}\left(\mathbb{P}^{n}(\mathbb{R}), \mathbb{Z} / 2 \mathbb{Z}\right)\right)
$$

of $H^{*}(X(\mathbb{R}), \mathbb{Z} / 2 \mathbb{Z})$.

Let $\tau$ be the tangent bundle of $X(\mathbb{R})$, let $\theta$ be the restriction to $X(\mathbb{R})$ of the tangent bundle of $\mathbb{P}^{n}(\mathbb{R})$, and let $v$ be the normal bundle of $X(\mathbb{R}) \subset \mathbb{P}^{n}(\mathbb{R})$. Since $\tau \oplus v \simeq \theta$, we get in terms of Stiefel-Whitney classes that $w(\tau) w(v)=w(\theta)$, hence $w(X(\mathbb{R}))=w(\tau)=w(\theta) w(v)^{-1}$. Since $w(\theta) \in R$ it is sufficient to show that $w(v)^{-1}$ is in $R$, but for this it is sufficient to show that $w(v) \in R$ (see [17, page 40]).

The normal bundle $v$ is of $\operatorname{rank} n-\operatorname{dim} X$ and algebraic, so

$$
w(v) \in \bigoplus_{k=1}^{n-\operatorname{dim} X} H_{\mathrm{alg}}^{k}(X(\mathbb{R}), \mathbb{Z} / 2 \mathbb{Z}) .
$$

The hypothesis $3 n \leq 4 \operatorname{dim} X$ implies that $n-\operatorname{dim} X \leq \operatorname{dim} X-n / 2$, so Corollary 2.4 gives that $w(v) \in R$.

Definition 2.6. We will say that an algebraic subset of $\mathbb{R}^{n}$ is projectively closed if it is also Zariski-closed in $\mathbb{P}^{n}(\mathbb{R})$.

Remark 2.7. By Tognoli's theorem ( $[21,22]$ ) (see also Nash's paper [18] for a weaker but influential result), each compact positive-dimensional smooth manifold $M$ is diffeomorphic to a nonsingular irreducible algebraic subset $A$ of $\mathbb{R}^{q}$ for some $q$. Taking the Zariski-closure of $A$ in $\mathbb{P}^{q}$ (as a real variety) and applying Hironaka's 
resolution of singularities [9], we see that $M$ is diffeomorphic to $X(\mathbb{R})$, where $X$ is a nonsingular closed subvariety of $\mathbb{P}^{r}$ for some $r$. Moreover $X(\mathbb{R}) \subset \mathbb{R}^{r}$, in other words, $X(\mathbb{R})$ is a nonsingular irreducible projectively closed algebraic subset of $\mathbb{R}^{r}$. Making use, if necessary, of general projections, we may assume $r=2 \operatorname{dim} M+1$.

Proof of Theorem 1.1. Let $M$ be a compact smooth manifold of dimension $m \geq 1$, and let $l$ be an arbitrary nonnegative integer.

(i) Clearly, $S^{l}$ can be embedded in $\mathbb{R}^{2 m+l}$ with trivial normal bundle. The rank of this bundle is $2 m$. By Whitney's theorem ([24]), there is a smooth embedding of $M$ into $\mathbb{R}^{2 m}$. Hence $M \times S^{l}$ admits a smooth embedding into $\mathbb{R}^{2 m+l}$, hence into $\mathbb{P}^{2 m+l}(\mathbb{R})$.

Now let $k$ be as in the theorem, and let $n, l$ be such that $2 m+2 l-2 k \geq n \geq$ $2 m+l$. Assume that $M \times S^{l}$ is not $n$-transcendental. Hence there exists a smooth embedding $e: M \times S^{l} \rightarrow \mathbb{P}^{n}(\mathbb{R})$ with $e\left(M \times S^{l}\right)=X(\mathbb{R})$ for some nonsingular closed subvariety $X$ of $\mathbb{P}^{n}$. Since $k \leq(m+l)-n / 2$,

$$
w_{i}\left(M \times S^{l}\right) \in e^{*}\left(H^{i}\left(\mathbb{P}^{n}(\mathbb{R}), \mathbb{Z} / 2 \mathbb{Z}\right)\right)
$$

for all $i \leq k$ by Corollary 2.4. Fixing a point $p \in S^{l}$, we now define $j: M \rightarrow$ $M \times S^{l}$ by $x \mapsto(x, p)$. Then $w_{i}(M)=j^{*}\left(w_{i}\left(M \times S^{l}\right)\right)$ for all $i \geq 0$ hence $w_{i}(M)$ is in $j^{*}\left(e^{*}\left(H^{i}\left(\mathbb{P}^{n}(\mathbb{R}), \mathbb{Z} / 2 \mathbb{Z}\right)\right)\right)$, hence a scalar multiple of $j^{*}\left(e^{*}\left(h^{i}\right)\right)$ where $h \in H^{1}\left(\mathbb{P}^{n}(\mathbb{R}), \mathbb{Z} / 2 \mathbb{Z}\right) \simeq \mathbb{Z} / 2 \mathbb{Z}$ is the class of the hyperplane. This contradicts the hypothesis, hence $M \times S^{l}$ is $n$-transcendental.

(ii) Suppose $n \geq 2 m+l+1$. According to Remark 2.7 we may assume that $M$ is a nonsingular irreducible projectively closed algebraic subset of $\mathbb{R}^{2 m+1}$. Applying a translation, we get $M$ contained in the open half-space

$$
\left\{\left(x_{1}, x_{2}, \ldots, x_{2 m+1}\right) \in \mathbb{R}^{2 m+1}: x_{2 m+1}>0\right\} .
$$

Define $\phi: M \times S^{l} \rightarrow \mathbb{R}^{2 m+l+1}$ by $\phi\left(\left(x_{1}, \ldots, x_{2 m+1}\right),\left(y_{1}, \ldots, y_{l+1}\right)\right)=\left(x_{1}, \ldots, x_{2 m}, x_{2 m+1} y_{1}, \ldots, x_{2 m+1} y_{l+1}\right)$.

One can check that the image $\phi\left(M \times S^{l}\right)$ is a projectively closed algebraic subset of $\mathbb{R}^{2 m+l+1}$, and $\phi: M \times S^{l} \rightarrow \phi\left(M \times S^{l}\right)$ is a biregular isomorphism of real algebraic sets ( $c f$. [13, Lemma 3.1] if desired).

\section{Equivariant cohomology}

Let $\Gamma=\operatorname{Gal}(\mathbb{C} / \mathbb{R})=\{1, \sigma\}$ be the Galois group of $\mathbb{C} / \mathbb{R}$. Recall, that $\Gamma$ acts on the set of complex points $X(\mathbb{C})$ of any real variety $X$, that this action is continuous for the Euclidean topology (in fact, it is anti-holomorphic), and that the set of fixed points under this action consists exactly of the set of real points $X(\mathbb{R})$ of the real variety $X$. Therefore, we get for any $\Gamma$-module $A$ (i.e., an abelian group $A$ with an 
action of $\Gamma)$, the equivariant cohomology $H^{*}(X(\mathbb{C}) ; \Gamma, A)$ of $X(\mathbb{C})$ by taking the equivariant sheaf cohomology (as defined in $[8$, Chapter $\mathrm{V}]$ ) of the equivariant sheaf associated to $A$ on the $\Gamma$-space $X(\mathbb{C})$, equipped with the Euclidean topology. For us the interesting cases are $A=\mathbb{Z} / 2 \mathbb{Z}$ and $A=\mathbb{Z}(i)$ (for any integer $i$ ), by which we mean that $A=\mathbb{Z}$ as an abelian group, and that $\Gamma$ acts on $A$ via $\sigma \cdot z=(-1)^{i} z$. It was V.A. Krasnov who first introduced the technique of equivariant cohomology into real algebraic geometry ([10]) and it has had many applications since then (see for example [7, 16, 19, 20,23]). For us, the following facts will be important.

Proposition 3.1. With notation as above, there is a spectral sequence

$$
E_{2}^{p q}=H^{p}\left(\Gamma, H^{q}(X(\mathbb{C}), A)\right) \Rightarrow H^{p+q}(X(\mathbb{C}) ; \Gamma, A),
$$

which is functorial in $X$ and $A$.

Proof. See [8, Theorem 5.2.1].

The second important fact uses natural homomorphisms

$$
\beta^{s, t}=\beta_{A}^{s, t}: H^{s}(X(\mathbb{C}) ; \Gamma, A) \rightarrow H^{t}(X(\mathbb{R}), \mathbb{Z} / 2 \mathbb{Z})
$$

defined for $A=\mathbb{Z} / 2 \mathbb{Z}$ or $\mathbb{Z}(i)$, which are functorial in $X$ (see for example [11]). The map $\beta_{A}^{s, t}$ is in fact the composition of the change of coefficients map

$$
H^{s}(X(\mathbb{C}) ; \Gamma, A) \rightarrow H^{s}(X(\mathbb{C}) ; \Gamma, \mathbb{Z} / 2 \mathbb{Z}),
$$

followed by the restriction map $H^{s}(X(\mathbb{C}) ; \Gamma, \mathbb{Z} / 2 \mathbb{Z}) \rightarrow H^{s}(X(\mathbb{R}) ; \Gamma, \mathbb{Z} / 2 \mathbb{Z})$, followed by the isomorphism $H^{s}(X(\mathbb{R}) ; \Gamma, \mathbb{Z} / 2 \mathbb{Z}) \simeq \bigoplus_{k=0}^{s} H^{k}(X(\mathbb{R}), \mathbb{Z} / 2 \mathbb{Z})([8$, Corollary of Proposition 5.4.1]), followed by the projection to the $t$ th term of the direct sum. (Indeed, $\beta_{A}^{s, t}$ is the zero map if $t>s$ ).

For us, the importance of the maps $\beta^{s, t}$ lies in the following. If $X$ is nonsingular and projective over $\mathbb{R}$, then for each $k \geq 0$ we have a cycle map

$$
\mathcal{Z}^{k}(X) \rightarrow H^{2 k}(X(\mathbb{C}) ; \Gamma, \mathbb{Z}(k))
$$

with the remarkable property (first proved by V.A. Krasnov in [12]) that not only the 'complex' cycle map

$$
\mathrm{cl}_{\mathbb{C}}: \mathcal{Z}^{k}(X) \rightarrow H^{2 k}(X(\mathbb{C}), \mathbb{Z}(k))
$$

factorizes through (3.2), via the natural map

$$
H^{2 k}(X(\mathbb{C}) ; \Gamma, \mathbb{Z}(k)) \rightarrow H^{2 k}(X(\mathbb{C}), \mathbb{Z}(k)),
$$


but that the real cycle map

$$
\operatorname{cl}_{\mathbb{R}}: \mathcal{Z}^{k}(X) \rightarrow H^{k}(X(\mathbb{R}), \mathbb{Z} / 2 \mathbb{Z})
$$

factorizes through (3.2) as well, via the map

$$
\beta^{2 k, k}: H^{2 k}(X(\mathbb{C}) ; \Gamma, \mathbb{Z}(k)) \rightarrow H^{k}(X(\mathbb{R}), \mathbb{Z} / 2 \mathbb{Z})
$$

defined above (see [12], or [23, Chapter IV] for a different approach).

From these remarkable facts we easily derive the following result.

Lemma 3.2. Let $f: X \rightarrow Y$ be a morphism of algebraic varieties such that

$$
f(\mathbb{C})^{*}: H^{2 k}(Y(\mathbb{C}) ; \Gamma, \mathbb{Z}(k)) \rightarrow H^{2 k}(X(\mathbb{C}) ; \Gamma, \mathbb{Z}(k))
$$

is surjective, then

$$
H_{\mathrm{alg}}^{k}(X(\mathbb{R}) ; \mathbb{Z} / 2 \mathbb{Z}) \subset f(\mathbb{R})^{*}\left(H^{k}(Y(\mathbb{R}), \mathbb{Z} / 2 \mathbb{Z})\right)
$$

Proof. By the above we have that

$$
H_{\mathrm{alg}}^{k}(X(\mathbb{R}) ; \mathbb{Z} / 2 \mathbb{Z}) \subset \beta^{2 k, k}\left(H^{2 k}(X(\mathbb{C}) ; \Gamma, \mathbb{Z}(k))\right) .
$$

By hypothesis,

$$
\beta^{2 k, k}\left(H^{2 d}(X(\mathbb{C}) ; \Gamma, \mathbb{Z}(k))\right)=\beta^{2 k, k}\left(f(\mathbb{C})^{*} H^{2 k}(Y(\mathbb{C}) ; \Gamma, \mathbb{Z}(k))\right) .
$$

Functoriality of $\beta^{2 k, k}$ implies that

$$
\begin{aligned}
\beta^{2 k, k}\left(f(\mathbb{C})^{*}\left(H^{2 k}(Y(\mathbb{C}) ; \Gamma, \mathbb{Z}(k))\right)\right) & =f(\mathbb{R})^{*}\left(\beta^{2 k, k}\left(H^{2 k}(Y(\mathbb{C}) ; \Gamma, \mathbb{Z}(k))\right)\right) \\
& \subset f(\mathbb{R})^{*}\left(H^{d}(Y(\mathbb{R}), \mathbb{Z} / 2 \mathbb{Z})\right) .
\end{aligned}
$$

Proof of Theorem 2.1. Let $f: X \rightarrow Y$ be as in the statement of the Theorem. Since

$$
f(\mathbb{C})^{*}: H^{q}(Y(\mathbb{C}), \mathbb{Z}) \rightarrow H^{q}(X(\mathbb{C}), \mathbb{Z})
$$

is an isomorphism for $q \leq r$, we have that the induced map

$$
H^{p}\left(\Gamma, H^{q}(Y(\mathbb{C}), \mathbb{Z}(i))\right) \rightarrow H^{p}\left(\Gamma, H^{q}(X(\mathbb{C}), \mathbb{Z}(i))\right)
$$

is an isomorphism for every $p \geq 0, q \leq r$ and $i \in \mathbb{Z}$. Therefore, the spectral sequence (3.1) tells us that

$$
f(\mathbb{C})^{*}: H^{j}(Y(\mathbb{C}) ; \Gamma, \mathbb{Z}(i)) \rightarrow H^{j}(X(\mathbb{C}) ; \Gamma, \mathbb{Z}(i))
$$


is an isomorphism for all $j \leq r$ and all $i \in \mathbb{Z}$. In particular,

$$
f(\mathbb{C})^{*}: H^{2 k}(Y(\mathbb{C}) ; \Gamma, \mathbb{Z}(k)) \rightarrow H^{2 k}(X(\mathbb{C}) ; \Gamma, \mathbb{Z}(k))
$$

is an isomorphism for $2 k \leq r$, and Lemma 3.2 then implies that

$$
H_{\text {alg }}^{k}(X(\mathbb{R}), \mathbb{Z} / 2 \mathbb{Z}) \subset f(\mathbb{R})^{*}\left(H^{k}(Y(\mathbb{R}), \mathbb{Z} / 2 \mathbb{Z})\right) .
$$

\section{References}

[1] S. Akbulut and H. King, Transcendental submanifolds of $\mathbf{R}^{n}$, Comment. Math. Helv. 68 (1993), 308-318.

[2] S. AkBulut and H. KING, Transcendental submanifolds of $\mathbb{R P}^{n}$, Comment. Math. Helv. 80 (2005), 427-432.

[3] J. BOCHNAK, M. BUCHNER and W. KUCHARZ, Vector bundles over real algebraic varieties, $K$-Theory 3 (1989), 271-298.

[4] J. Bochnak, M. Coste and M.-F. Roy, "Real Algebraic Geometry", Ergebnisse der Mathematik und ihrer Grenzgebiete (3), Vol. 36, Springer-Verlag, Berlin, 1998.

[5] J. BOCHNAK and W. KUCHARZ, On homology classes represented by real algebraic varieties, In: "Singularities Symposium-Łojasiewicz 70" (Kraków, 1996; Warsaw, 1996), Banach Center Publ., Vol. 44, Polish Acad. Sci., Warsaw, 1998, 21-35.

[6] A. Borel and A. HAefliger, La classe d'homologie fondamentale d'un espace analytique, Bull. Soc. Math. France 89 (1961), 461-513.

[7] A. Degtyarev, I. Itenberg and V. Kharlamov, "Real Enriques Surfaces", Lecture Notes in Mathematics, Vol. 1746, Springer-Verlag, Berlin, 2000.

[8] A. Grothendieck, Sur quelques points d'algèbre homologique, Tôhoku Math. J. 9 (1957), 119-221.

[9] H. HIRONAKA, Resolution of singularities of an algebraic variety over a field of characteristic zero. I, II, Ann. of Math. 79 (1964), 109-203; ibid. 79 (1964), 205-326.

[10] V. A. KRASNOV, Harnack-Thom inequalities for mappings of real algebraic varieties, Izv. Akad. Nauk SSSR Ser. Mat. 47 (1983), 268-297 (Russian); English transl., Math. USSRIzv. 22 (1984), 247-275.

[11] V. A. KRASNOv, Characteristic classes of vector bundles on a real algebraic variety, Izv. Akad. Nauk SSSR Ser. Mat. 55 (1991), 716-746 (Russian); English transl. Math. USSR-Izv. 39 (1992), 703-730.

[12] V. A. KRASNOV, On the equivariant Grothendieck cohomology of a real algebraic variety and its application, Izv. Ross. Akad. Nauk Ser. Mat. 58 (1994), 36-52 (Russian); English transl. Russian Acad. Sci. Izv. Math. 44 (1995), 461-477.

[13] W. KUCHARZ, Transcendental submanifolds of projective space, Comment. Math. Helv. 84 (2009), 127-133.

[14] M. E. LARSEN, On the topology of complex projective manifolds, Invent. Math. 19 (1973), 251-260.

[15] R. LAZARSFeld, "Positivity in Algebraic Geometry", I, Ergebnisse der Mathematik und ihrer Grenzgebiete, 3, Folge, Vol. 48, Springer-Verlag, Berlin, 2004.

[16] F. MAngolte and J. VAN HAMEL, Algebraic cycles and topology of real Enriques surfaces, Compositio Math. 110 (1998), 215-237.

[17] J. W. Milnor and J. D. StAShefF, "Characteristic Classes", Annals of Mathematics Studies, Vol. 76, Princeton University Press, Princeton, N.J., 1974.

[18] J. NASH, Real algebraic manifolds, Ann. of Math. 56 (1952), 405-421. 
[19] V. V. NikUlin and R. Sujatha, On Brauer groups of real Enriques surfaces, J. Reine Angew. Math. 444 (1993), 115-154.

[20] R. SiLHOL, "Real Algebraic Surfaces", Lecture Notes in Mathematics, Vol. 1392, SpringerVerlag, Berlin, 1989.

[21] A. Tognoli, Su una congettura di Nash, Ann. Scuola Norm. Sup. Pisa (3) 27 (1973), $167-185$.

[22] A. Tognoli, Une remarque sur les approximations en géométrie algébrique réelle, $\mathrm{C} . \mathrm{R}$. Acad. Sci. Paris Sér. I Math. 296 (1983), 745-747.

[23] J. VAN HAMEL, "Algebraic Cycles and Topology of Real Algebraic Varieties", CWI Tract, Vol. 129, Stichting Mathematisch Centrum, Amsterdam, 2000.

[24] H. Whitney, The self-intersections of a smooth n-manifold in 2n-space, Ann. of Math. 45 (1944), 220-246.

Department of Mathematics and Statistics University of New Mexico

Albuquerque, NM 87131, USA

kucharz@math.unm.edu

K.U. Leuven

Departement Wiskunde

Celestijnenlaan 200B

B-3001 Leuven (Heverlee), Belgium

vanhamel@member.ams.org 